\title{
VISIBILITY DIAGRAMS AND EXPERIMENTAL STRIPE STRUCTURES IN THE QUANTUM HALL EFFECT
}

\author{
Yvon Georgelin $^{a}$, Thierry Masson ${ }^{b}$ and Jean-Christophe Wallet ${ }^{a}$ \\ ${ }^{a}$ Groupe de Physique Théorique, Institut de Physique Nucléaire \\ F-91406 ORSAY Cedex, France \\ ${ }^{b}$ Laboratoire de Physique Théorique (U.M.R. 8627), Université de Paris-Sud \\ Bât. 211, F-91405 ORSAY Cedex, France
}

\begin{abstract}
We analyze various properties of the visibility diagrams that can be used in the context of modular symmetries and confront them to some recent experimental developments in the Quantum Hall Effect. We show that a suitable physical interpretation of the visibility diagrams which permits one to describe successfully the observed architecture of the Quantum Hall states gives rise naturally to a stripe structure reproducing some of the experimental features that have been observed in the study of the quantum fluctuations of the Hall conductance. Furthermore, we exhibit new properties of the visibility diagrams stemming from the structure of subgroups of the full modular group.
\end{abstract}

(June 1999)

LPT-99/27 


\section{INTRODUCTION}

The Quantum Hall Effect (QHE) is a remarquable phenomenon occuring in a twodimensional electron gas in a strong magnetic field at low temperature [1]. Since the discovery of the quantized integer [2] and fractional [3] Hall conductivity, the QHE has been an intensive area of theoretical and experimental investigations. The pioneering theoretical contributions [4] analyzing the basic features of the hierarchy of the Hall plateaus have triggered numerous works aiming to provide a better understanding of the underlying properties governing the complicated phase diagram associated with the quantum Hall regime together with the precise nature of the various observed transitions between plateaus and/or focusing on a characterization of a suitable theory.

It has been realized for some time that modular symmetries may well be of interest to understand more deeply salient features of the QHE. For instance, it has been suggested [5], [6a-b] that some properties of the phase diagram may be explained in terms of modular group transformations. At the present time, a fully satisfactory microscopic or effective theory for the QHE, from which the relevant modular symmetry (if any) would come out, is still lacking. This has motivated further studies focalized on the derivation of general constraints on the phase diagram coming for the full modular group or some of its subgroups [6a-b].

Some time ago, we have shown that a special subgroup of the full modular group, namely the group $\Gamma(2)$, can be used to derive a model for a classification of integer as well as fractional Hall states $[7 a-b]$. We have further shown that the constraints stemming from $\Gamma(2)$ on physically admissible $\beta$-functions [8] give rise to a global phase diagram as well as crossover in the various observed transitions which are in good agreement with the present experimental observations. Here, it is worth recalling that the classification based on the $\Gamma(2)$ symmetry [7a-b], which refines the Jain and Haldane ones and can be somehow viewed as a kind of generalization of the law of the corresponding states proposed in [9], seems to reproduce successfully the observed hierarchical structure of the Hall states. This construction involves two important building blocks (in addition to the action of the group $\Gamma(2)$ itself) called the visibility diagrams. Basically, these diagrams, inherited from theoretical studies in arithmetics and rigidely linked to the structure of $\Gamma(2)$, have been shown to encode a great amount of information on the experimentaly observed global organization of the quantum Hall states.

Recently, some new experiments on the mesoscopic conductance in the quantum Hall regime in silicon MOSFETs have been performed [10]. The main result is that the extrema of the conductance fluctuations spread on linear trajectories in the gate voltage $V_{g}$-magnetic field $B$ plane. These lines are parallels to lines of constant filling factor $\nu=p$ (with $p=0,1,2, \ldots)$. Moreover, a clear stripe structure can be observed [10] on this plane.

The purpose of this note is to analyze more deeply various properties of the visibility diagrams (therefore extending our previous investigations [7b])as well as to confront them to the present experimental situation for the QHE. We show that a suitable physical interpretation of the visibility diagrams that permits one to describe successfully the observed architecture of the Quantum Hall states gives rise naturally to a stripe structure that reproduces some of the experimental features of the stripe structure of the conductance fluctuations in the $V_{g}-B$ plane. This is presented in section 2 where the interesting role 
played by the visibility diagrams in the description of some of the physics of the Quantum Hall regime is emphasized. Furthermore, we exhibit in section 3 new properties of these diagrams stemming from the structure of subgroups of the full modular group. Finally, we summarize the results and conclude.

\section{VISIBILITY DIAGRAMS AND STRIPE STRUCTURES}

Let us first recall the essential features of $\Gamma(2)$ that will be needed in the following analyzis. The group $\Gamma(2)$ is the set of transformations $G$ acting on the upper-half complex plane $\mathcal{P}$ which can be written as

$$
G(z)=\frac{(2 s+1) z+2 n}{2 r z+(2 k+1)}, \quad k, n, r, s \in Z
$$

where

$$
(2 s+1)(2 k+1)-4 r n=1 \text { unimodularity condition }
$$

and $z \in \mathcal{P}(\operatorname{Im} z>0)$. The two generators of $\Gamma(2)$ are defined by

$$
T^{2}(z)=z+2, \quad \Sigma(z)=\frac{z}{2 z+1}
$$

Now to construct from $\Gamma(2)$ a model for the classification of the quantum Hall states identify first the complex coordinate $z$ with the filling factor $\nu=p / q$. Then, as we have shown in [7a], for a given (fixed) even denominator metallic state $\lambda=\frac{2 s+1}{2 r}$, the hierarchy of the (liquid) odd denominator states surrounding this metallic state is obtained from the images $G_{n, k}^{\lambda}(0)$ and $G_{n, k}^{\lambda}(1)$ of 0 and 1 by the family of transformations $G_{n, k}^{\lambda} \in \Gamma(2)$ where $\lambda=\frac{2 s+1}{2 r}$ holds and $n, k$ are constrained by the unimodularity condition $(2)$.

The action of $\Gamma(2)$ on the filling factor $\nu=p / q$ can be visualized with the help of graphical representations called visibility diagrams whose construction is now summarized (for a detailed construction see $[7 \mathrm{~b}]$ ). Consider a two-dimensional square lattice whose vertices are indexed by a couple of positive (or zero) relatively prime integers $(q, p)$. Since $\Gamma(2)$ preserves the parity of the denominator of any rational fraction, there are actually two ways for organizing the vertices pertaining to this lattice, depending whether the denominator is even or odd.

Consider first the case where it is even and choose therefore a given $\lambda=\frac{2 s+1}{2 r}$ as a starting vertex. Then, it is not difficult to realize that $G_{n, k}^{\lambda}(0)$ and $G_{n, k}^{\lambda}(1)$ which in the present framework label the Hall plateaus surrounding the even denominator (metallic) Hall state corresponding to $\lambda$ are all located on two parallel straigh lines forming an unbounded left-ended stripe surrounding the vertex $\lambda$. Finally, the application of a similar process to all even denominator fractions gives rise to a collection of non-overlapping stripes as depicted on fig.1, each stripe corresponding therefore to a vertex with even denominator. This visibility diagram, hereafter called even diagram, involves naturally the Jain hierarchy which corresponds to the stripe associated with $\lambda=1 / 2$ as it can be easily realized by computing the successive values for $G_{n, k}^{1 / 2}(0)$ and $G_{n, k}^{1 / 2}(0)$ using (1) and (2). It is worth recalling some experimental results performed in [11-13] on metal-insulator transitions. 
It appears that the corresponding phase diagrams (see e.g. fig.2 of ref.[11] and fig.2 of ref.[12]; see also a recent result reported in [13]) exhibit a stripe structure which bears some similarity with the stripe structure occuring on the even diagram when, anticipating what will be done in a while, the $(q, p)$ plane is identified with the magnetic field-charge carrier density planeł. This can be easily illustrated by selecting from fig.1 the relevant stripes which corresponds here to $\lambda=(2 s+1) / 2$ as shown on fig.2 to be compared to the fig.3 of ref.[13]. We close this part of the discussion by noting that an experimental phase diagram of the integer QHE (charge carrier density $\left(\sim V_{g}\right)$ versus magnetic field) has been explicitely obtained very recently in [14] suggesting that the vertical leftmost half stripe that is involved in the even diagram might well be associated with some insulator state. In fact, provided the above interpretation of the $(q, p)$ plane is actually correct, this vertical stripe (indexed by "1/0") might correspond to the type I insulator indicated in [14], whose properties are different from the type II insulator.

The second diagram that can be constructed, hereafter called the odd diagram, can be readily obtained by using a well-known theorem in arithmetics which states that for any relatively prime integers $q$ and $p$ there exist (necessarily) prime integers $a$ and $b$ such that

$$
q b-p a= \pm 1
$$

Then, for any $(q, p)$ vertex of the lattice with $q$ odd, associate the set of points $(a, b)$ satisfying this relation. It is easy to realize that these points are located on two parallel straight lines forming a stripe surrounding the $(q, p)$ vertex as depicted on fig. 3 . We then obtain stripes for any odd denominator fraction $q / p$. Notice that the stripes can overlap, contrary to what happens for the even diagram *.

We are now in position to show that this latter diagram encodes interesting information concerning some recent experimental results related to the quantum Hall fluctuations of the conductance that have been reported in [10]. The main result of this study on the mesoscopic conductance in the Quantum Hall regime in Silicon MOSFET is that the extrema for the conductance fluctuations spread on linear trajectories in the $V_{g}$ - $B$ plane parallel to constant (integer) filling factor. Namely, the following relation

$$
\frac{C}{e} \frac{\partial V_{g}}{\partial B}=n \frac{e}{h}, n \text { integer }
$$

in which $\frac{C}{e}=\frac{\partial \rho}{\partial V_{g}}$ is assumed to hold ( $\rho$ denotes the electron density and the constant $\frac{C}{e}=8.610^{11} \mathrm{~cm}^{-2} \mathrm{~V}^{-1}$ in [10]) has been found to be verified within a few percent of accuracy. Moreover, a stripe structure in the $V_{g}-B$ plane has been observed, as shown on fig.4 (taken from the fig.2 of [10]).

In order to confront some of these experimental results to the present $\Gamma(2)$ framework, we have first to exhibit a possible relation between the visibility diagram, the gate voltage and the applied magnetic field. This proceeds as follows. First, recall that the filling factor $\nu=\frac{p}{q}$ can be expressed from its very definition as

$$
\nu=\frac{N_{c}}{N_{\Phi}}
$$

\footnotetext{
$\mp$ at least for not too small charge carrier density

* It is not dififcult to see that the Haldane hierarchy is involved in the odd visibility diagram.
} 
in which $N_{c}$ is the number of charge carriers (the electrons in the present case) and $N_{\Phi}=B S \frac{h}{e^{2}}$ ( $S$ is the device area) is the number of unit flux. Then observe that the action of the operator $T^{2}$ (3.a), a Landau shift type operator, on any vertex $(q, p)$ of the visibility diagram gives rise to a vertical shift $\left(T^{2}:(q, p) \rightarrow(q, p+2 q)\right)$ whereas the action on any vertex of $\Sigma$ (3.b) which is the flux attachement operator produces a horizontal shift $(\Sigma:(q, p) \rightarrow(q+2 p, p))$. Next, observe that the charge carrier density happens to be proportional to the gate voltage $V_{g}$ in the experiments reported in [10] and that the flux attachement is obtained by magnetic field variation. Bringing this all together, this suggests to identify naturally (up to dimensionful factors) the horizontal (resp. vertical) axis on the visibility diagram with the $B$-axis (resp. $V_{g}$-axis).

Owing to the above identification, it is now possible to perform a comparison between the stripe structure occuring in the $V_{g}-B$ plane observed in [10] (represented by the grey areas associated with the plateaus) and the one stemming from the odd diagram. These structures are depicted respectively on fig.4 and fig.5. For the sake of clarity, we have only represented on fig. 5 the stripes corresponding to the integer filling factors considered in [10]. We observe a good qualitative agreement between both structures. Each grey area on fig. 4 corresponding to a given (integer) plateau appears to be bounded by two parallel straight lines (at least in the considered range for $V_{g}$ and $B$ ). Taking into account the method for constructing the odd diagram, it seems natural to identify each grey area associated with a given integer filling factor with the corresponding stripe on fig.5.

If this latter identification is physically correct, it is possible to obtain from the odd diagram further information on the Hall conductance as a function of $V_{g}$ (uppermost onset of fig.4) by adapting to the present situation, for which $B$ is fixed while $V_{g}\left(N_{c}\right)$ varies, the argument that we used in [7b] to obtain a resistivity plot (Hall resistivity versus $B$ ) fitting well with the experimental plot. This is straighforwardly achieved by simply assuming that the vertical width of any stripe on fig.5, defined by the intercept of any vertical line with that stripe, is proportional to the width of the corresponding plateau. The subsequent analysis is then very similar to the one that we described in [7b]. We have found that the resulting conductivity- $V_{g}$ plot agrees qualitatively with the one depicted on fig. 4 .

At this point, the analysis suggests that the proposed physical interpretation of the odd diagram is consistent with the experimental observations corresponding to the integer QHE. Proving that this diagram encodes some relevant properties of both integer and fractional QHE (giving therefore some global information on the phase diagrams) would require further confrontation with experiments exploring the fractional quantum Hall regime (and/or for higher magnetic field). In this regime, it is obvious from the very construction of the odd diagram that one (experimentaly testable) prediction of the present scheme is the occurence of branching tree-like structures [7b] among the stripes in the $V_{g}-B$ plane similar to the one appearing on fig.3.

Let us now consider the quantum Hall fluctuations. Recall that these fluctuations are observed in the transition regions between plateaus which narrow as the temperature decreases while the fluctuations grow and sharpen. Obviously, a full confrontation of the experimentaly verified relation (5) to the present $\Gamma(2)$ framework would require to have at hand a detailled model for the quantum Hall fluctuations $\dagger$ providing a satisfactory

$\dagger$ Note that relation (5) seems to contradict the predictions stemming from the non-interacting models. 
explanation for the origin of the observed behaviour. Nevertheless, keeping in mind the above analysis of the stripe structures, it seems plausible to conjecture that, for a given transition $\nu=n_{1} \rightarrow \nu=n_{2}, n_{1,2} \in N$ (extending to $n_{1,2} \in Q$ if the present scheme applies to the fractional QHE), the directions of the two coexisting families of straight lines involving the extrema of the fluctuations (which are observed in [10] for the transitions $0 \rightarrow 1,1 \rightarrow 2,2 \rightarrow 3,3 \rightarrow 4)$ are given by the directions defined by the corresponding stripes involved in that transition.

\section{MORE ON VISIBILITY DIAGRAMS AND DISCUSSION}

From the above analysis, it appears that the visibility diagrams may well encode some physically relevant features of the QHE which motivates a deeper investigation of mathematical properties underlying their structure. This is what we consider now.

First, we point out that the odd diagram is in fact the superposition of two "more elementary" visibility diagrams. To see that, consider separately odd and even numerator filling factors (with odd denominator) and apply the method for constructing the odd visibility diagram that has been described in section 2. Doing this, one obtains the two new diagrams represented on fig.6 and fig.7 corresponding respectively to odd and even numerator filling factors (hereafter called respectively odd/odd and even/odd diagrams). Then, it can be easily realized that the superposition of these two latter diagrams gives rise to the odd visibility diagram. Note that the stripes appearing in these two diagrams do not overlap as it is the case for the even diagram. Furthermore, observe that this latter diagram is related to the even/odd diagram through a symmetry around the $p=q$ axis which corresponds to the action of an operation belonging to the full modular group $\Gamma(1)$ but not to $\Gamma(2)$.

Let us study more closely the action of modular transformations pertaining to $\Gamma(1)$ on the even, even/odd and odd/odd diagrams. Some remarks are in order. On one hand, it can be easily seen that the action of any $G \in \Gamma(1)$ preserves the arithmetic relation (4) ruling the whole construction of these diagrams. As a consequence, the action of $\Gamma(1)$ maps the stripe structure of each of the diagrams into another one or possibly a sub-structure. On the other hand, any $G \in \Gamma(2)$ maps each of these three diagrams into itself simply because $\Gamma(2)$ preserves the even or odd character of both numerator and denominator involved in the filling factor. In other words, the whole structure of each diagram remains invariant under $\Gamma(2)$. In fact, it appears that $\Gamma(2)$ is the largest subgroup of $\Gamma(1)$ leaving invariant each of the 3 diagrams. To see that, consider the action of the coset group $\Gamma(1) / \Gamma(2)$ on these diagrams. It is known in the mathematical litterature [15] that this coset group involves 6 elements whose corresponding representatives in $\Gamma(1)$ can be choosen as:

$$
\begin{array}{r}
I=\left(\begin{array}{ll}
1 & 0 \\
0 & 1
\end{array}\right), \quad U=\left(\begin{array}{ll}
1 & 1 \\
0 & 1
\end{array}\right), \quad V=\left(\begin{array}{cc}
0 & -1 \\
1 & 0
\end{array}\right) \\
W=\left(\begin{array}{ll}
1 & 0 \\
1 & 1
\end{array}\right), \quad P=\left(\begin{array}{cc}
0 & -1 \\
1 & 1
\end{array}\right), \quad P^{2}=\left(\begin{array}{cc}
-1 & -1 \\
1 & 0
\end{array}\right)
\end{array}
$$

from which it can be verified that any of the 6 coset group elements maps a visibility diagram into a sub-diagram included in another diagram. Finally, using (6) together 
with the definition of $\Gamma(2)$, it is easy to prove that the even and odd diagrams (depicted respectively on fig. 1 and fig.2) are invariant under the action of another subgroup of $\Gamma(1)$ generated by $U$ given by (6b) and $\Gamma(2)$. This subgroup is nothing but $\Gamma_{0}(2)$, which has been proposed [6b] (see also second of ref. [6a]) as another candidate for a discrete symmetry group underlying the physics of the QHE.

Note that the constraints from $\Gamma_{0}(2)$ on the renormalization group flow in a twoparameter scaling framework have been examined in [6b]. The resulting flow diagram (phase diagram) has been shown to exhibit a specific feature. In fact, consistency with the present experimental observations requires the occurence for the $0 \rightarrow 1$ transition $\dagger$ of a critical point at $\sigma_{x y}=\sigma_{x x}=1 / 2$ which appears as a pole of the corresponding $\beta$-function. This stemms from the existence of a fixed point of $\Gamma_{0}(2)$ in its fundamental domain at $z_{0}=(1+i) / 2$ (recall that in this framework one has $z=\sigma_{x y}+i \sigma_{x x}$ which parametrizes the conductivity plane). The situation is different in the $\Gamma(2)$ case, as shown recently in [8]: There is no critical point showing up as a pole (at finite distance in the conductivity plane) of the corresponding $\beta$-function but consistency with the two-parameter scaling hypothesis seems to require the occurence in each allowed transition of a temperature-independant point that might be identified with the crossing point appearing in the crossover of the observed transitions [8].

Let us summarize the results involved in this paper. We have analyzed various properties of the visibility diagrams which are related to the modular symmetries. In particular, we have shown that the observed structures occuring in the (gate voltage-magnetic field) data, at least in the integer Quantum Hall regime, may well be encoded in one visibility diagram, namely the odd one. We have also indicated an experimental way to test its possible relevance to the fractional QHE. Furthermore, we have conjectured that for a given transition $\nu=n_{1} \rightarrow \nu=n_{2}, n_{1,2} \in Q$ the directions of the two coexisting families of straight lines involving the extrema of the fluctuations ( observed in [10] for the transitions $0 \rightarrow 1,1 \rightarrow 2,2 \rightarrow 3,3 \rightarrow 4)$ are given by the directions defined by the corresponding stripes of the odd diagram involved in that transition.

$\dagger$ Recall that, as usual, the whole flow diagram is obtained by applying successive $\Gamma_{0}(2)$ transformations to the $0 \rightarrow 1$ "template" transition 


\section{REFERENCES}

1) The Quantum Hall Effect, 2nd ed., R.E. Prange and S.M. Girvin eds. (SpringerVerlag, New-York) 1990. See also Perspectives in Quantum Hall Effect, S.D. Sorma and A. Pinczuk eds. (Wiley, New-York) 1997.

2) K. von Klitzing, G. Dorda and M. Pepper, Phys. Rev. Lett. 45 (1980) 494.

3) D.C. Tsui, H.L. Störmer and A.C. Gossard, Phys. Rev. Lett. 48 (1982) 1559.

4) R.B. Laughlin, Phys. Rev. Lett. 50 (1983) 1385; F.D. Haldane, Phys. Rev. Lett. 51 (1983) 605; B.I. Halperin, Phys. Rev. Lett. 52 (1984) 1583.

5) C.A. Lütken and G.G. Ross, Phys. Rev. B45 (1992) 11837, Phys. Rev. B48 (1993) 2500 .

6a) C.A. Lütken, Nucl. Phys. B396 (1993) 670; C.P. Burgess and C.A. Lütken, On the implication of discrete symmetries for the $\beta$-function of Quantum Hall System, cond-mat/9812396 and references therein. See also E. Fradkin and S. Kivelson, Nucl. Phys. B474 (1996) 543.

6b) B.P. Dolan, J. Phys. A: Math. Gen. 32 (1999) L243; B.P. Dolan, Modular invariance, universality and crossover in the QHE, cond-mat/9809294.

7a) Y. Georgelin and J.C. Wallet, Phys. Lett. A224 (1997) 303.

7b) Y. Georgelin, T. Masson and J.C. Wallet, J. Phys. A:Math. Gen. 30 (1997) 5065.

8) Y. Georgelin, T. Masson and J.C. Wallet, $\Gamma(2)$ modular symmetry, renormalization group flow and the Quantum Hall Effect, cond-mat/9906193.

9) S. Kivelson, D.H. Lee and S.C. Zhang, Phys. Rev. B46 (1992) 2223.

10) D.H. Cobden, C.H. Barnes and C.J.B. Ford, Quantum Hall Fluctuations and evidence for charging in the Quantum Hall Effect, cond-mat/9902154.

11) S.V. Kravchenko, W. Mason and J.E. Furneaux, Phys. Rev. Lett. 75 (1995) 910.

12) S.V. Kravchenko et al., Phys. Rev. B51 (1995) 7038.

13) M. Hilke et al., experimental phase diagram of the integer quantized Hall effect, cond-mat/9906212.

14) D.N. Sheng and Z.Y. Weng, phase diagram of the integer Quantum Hall Effect, cond-mat/9906261.

15) See in D. Mumford, Tata Lectures on Theta functions, vol. I-III, (Birkhauser, Boston, Basel, Stuttgart) 1983. 


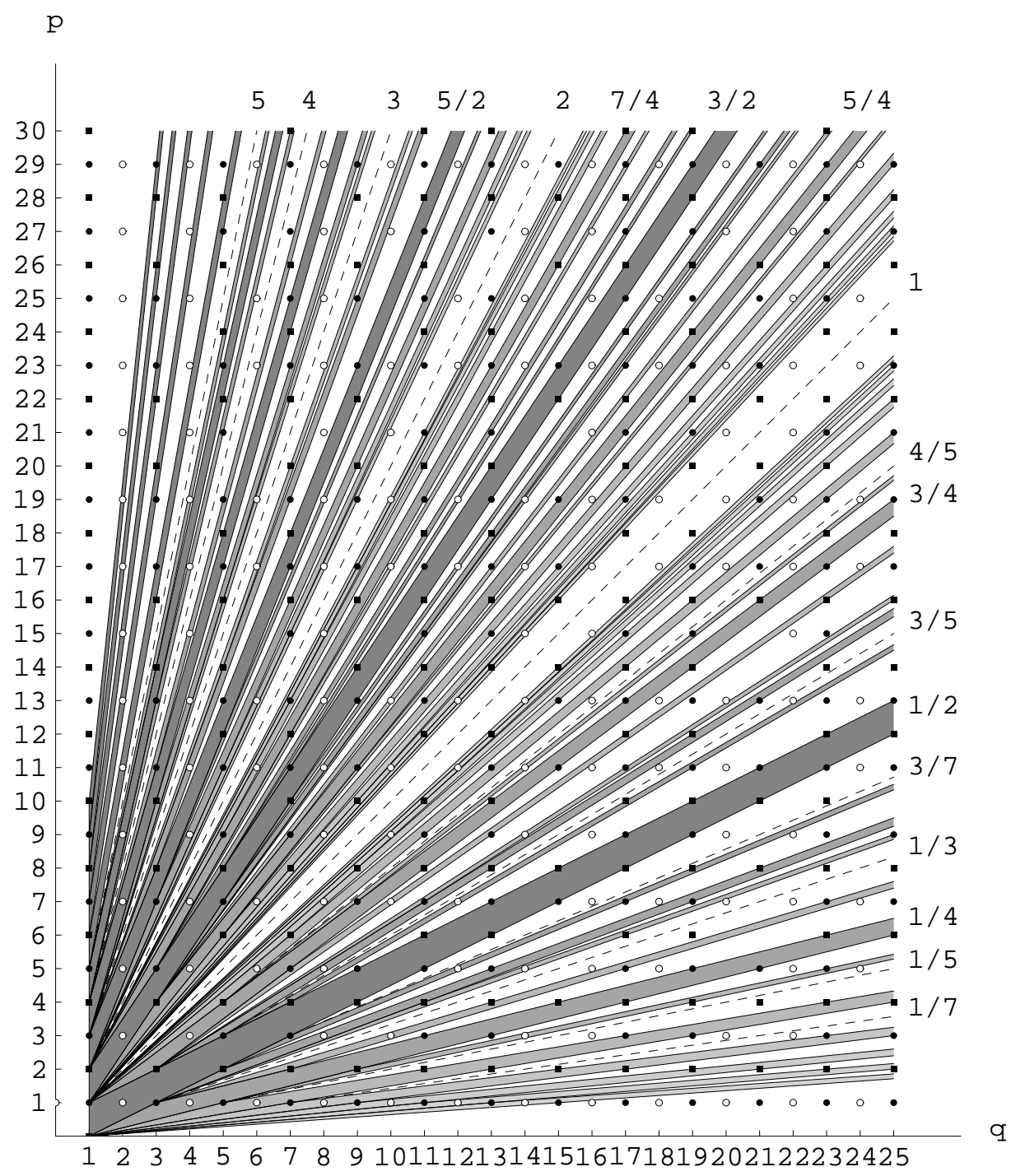

Figure 1: Even visibility diagram. The stripe indexed by $1 / 2$ corresponds to the Jain hierarchy. 


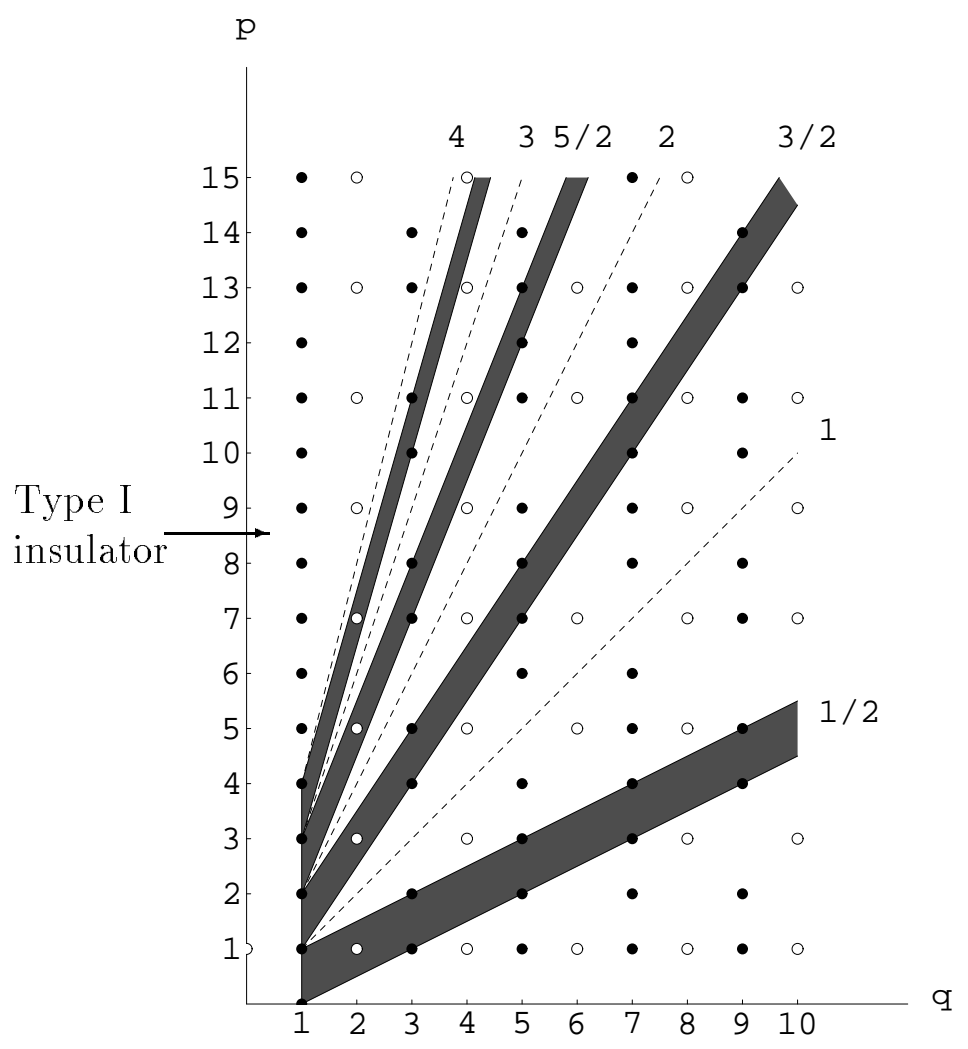

Figure 2: Selected stripes indexed by $\frac{2 s+1}{2}$, s integer. The comparison with fig.3 of [13] suggests to identify the vertical (half) stripe indexed by $q=0$ and $p=1$ with the type I insulator as observed in [13]. 


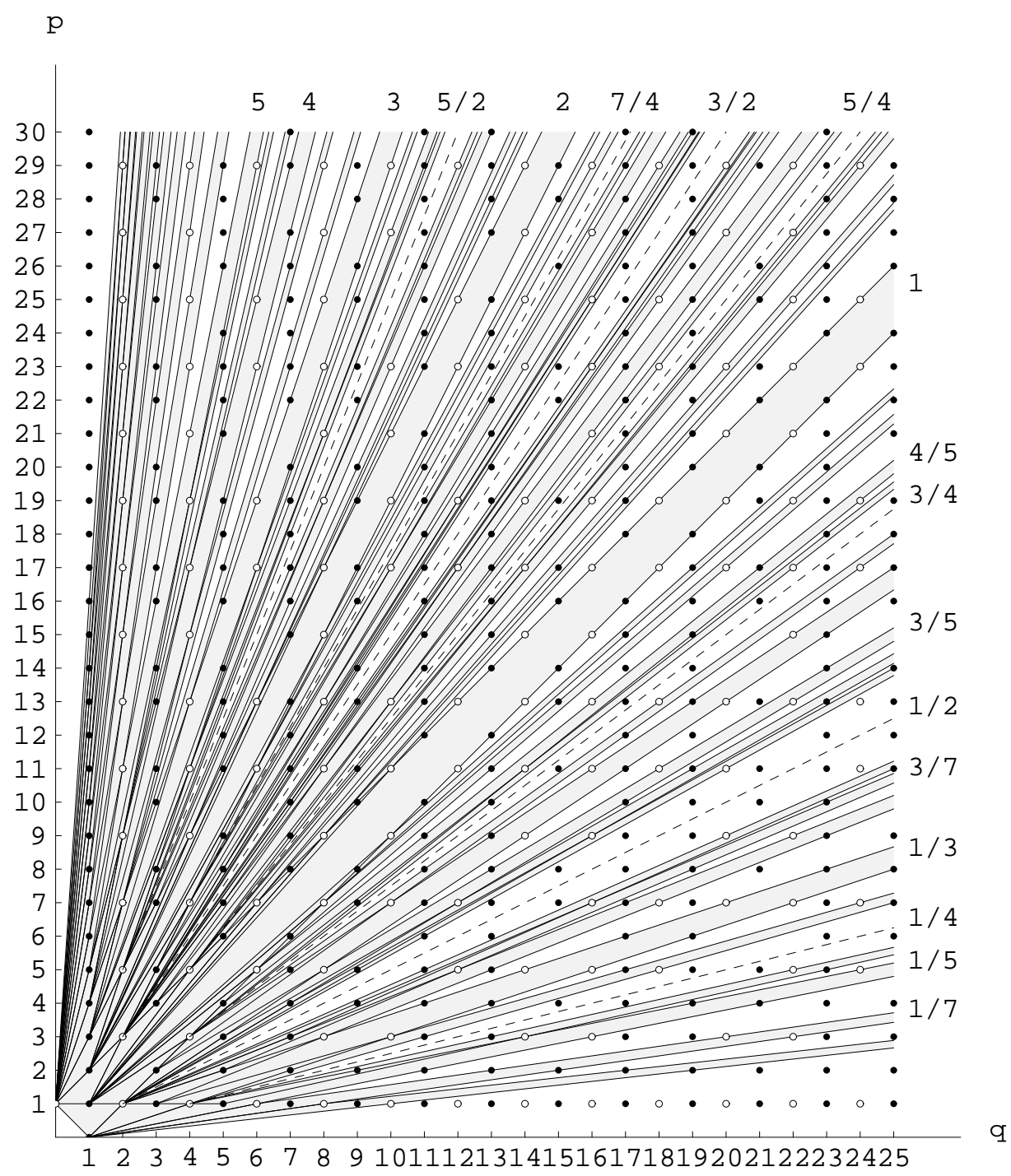

Figure 3: Odd visibility diagram. In [8], a numerical simulation based on information encoded in this diagram was performed for the $\rho_{x y}$ versus $B$ plot. 


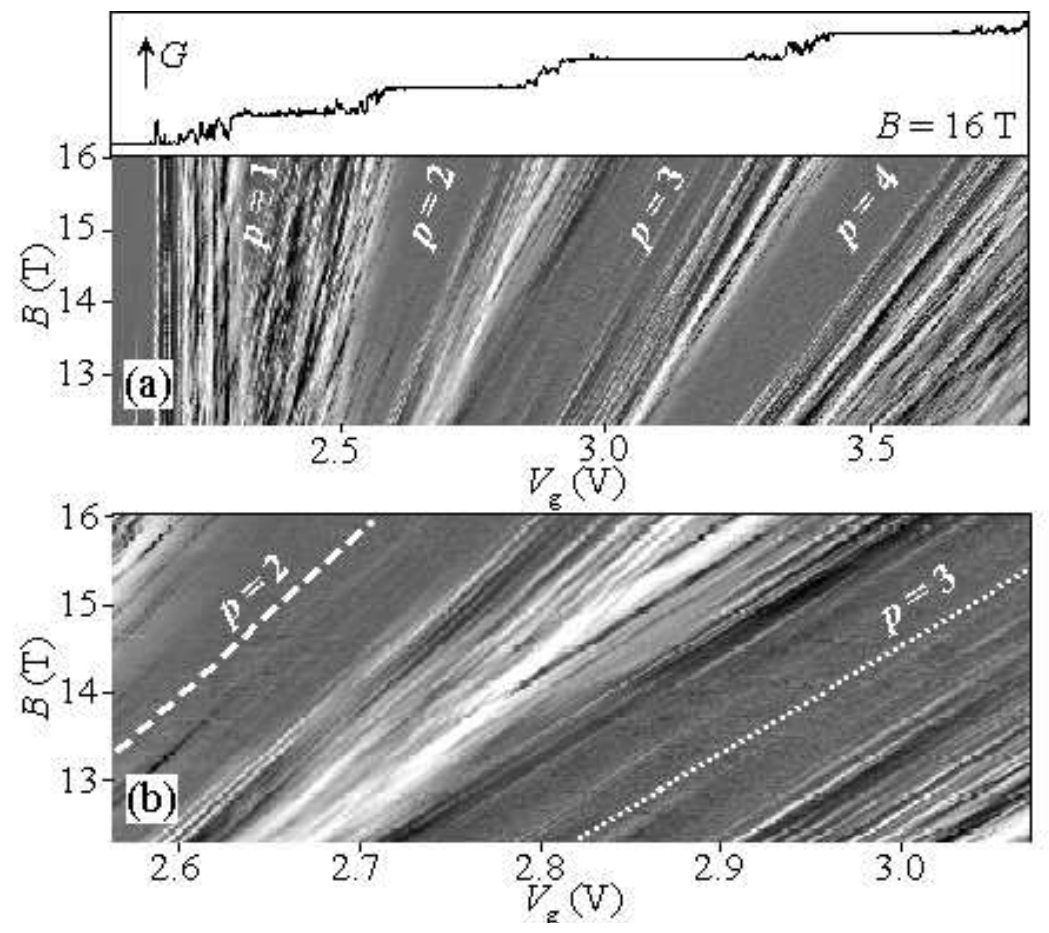

Figure 4: Grayscale plot of conductance with a smooth background substracted (lighter $=$ smaller $G$, where $G$ is the Hall conductance) taken from [15]. $p$ denotes the filling factor. 


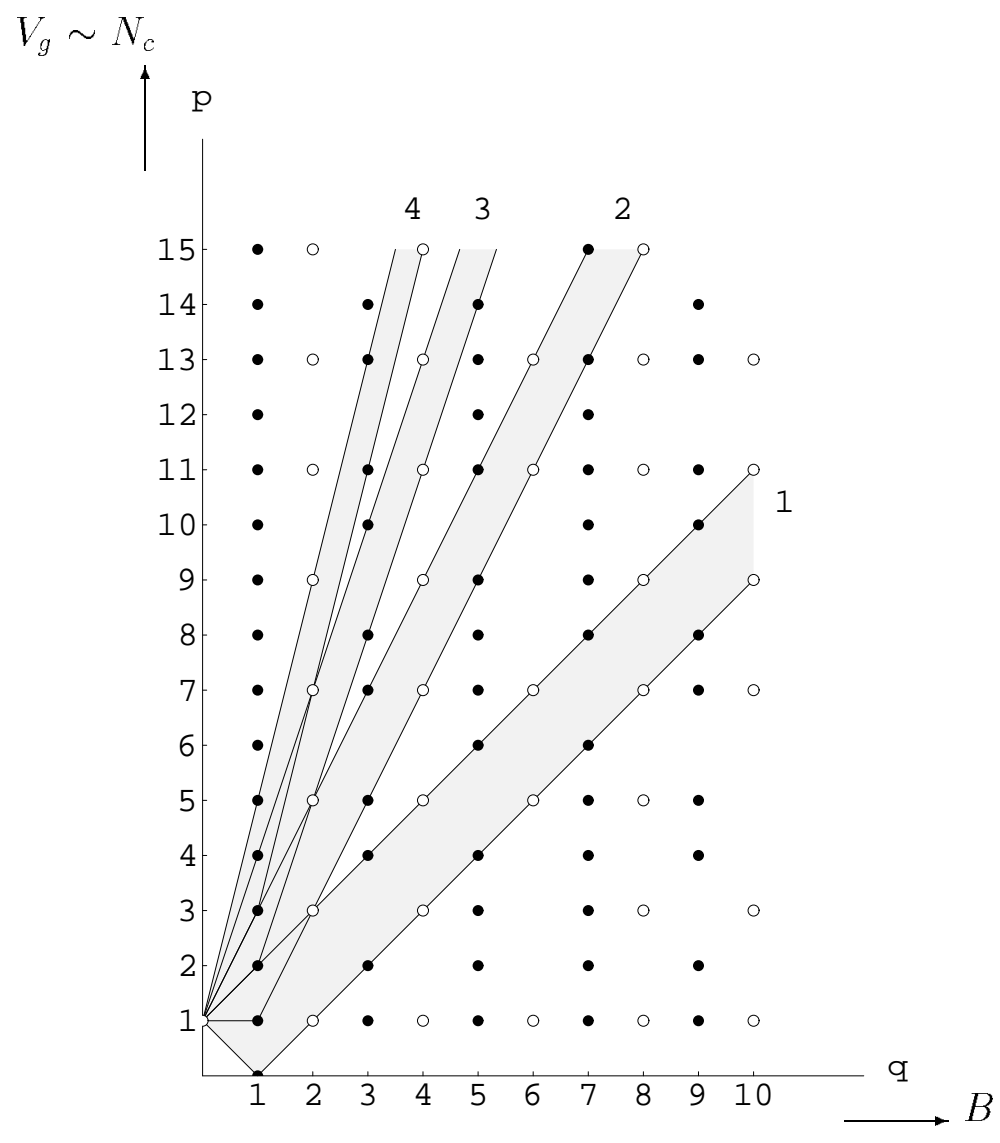

Figure 5: Selected stripes from the odd diagram corresponding to their experimental counterpart depicted in fig.4. Notice that the horizontal (resp. vertical) axis is associated with $B\left(\right.$ resp. $V_{g}$ ) so that a direct comparison to fig. 4 can be done after the axis here been permuted. 


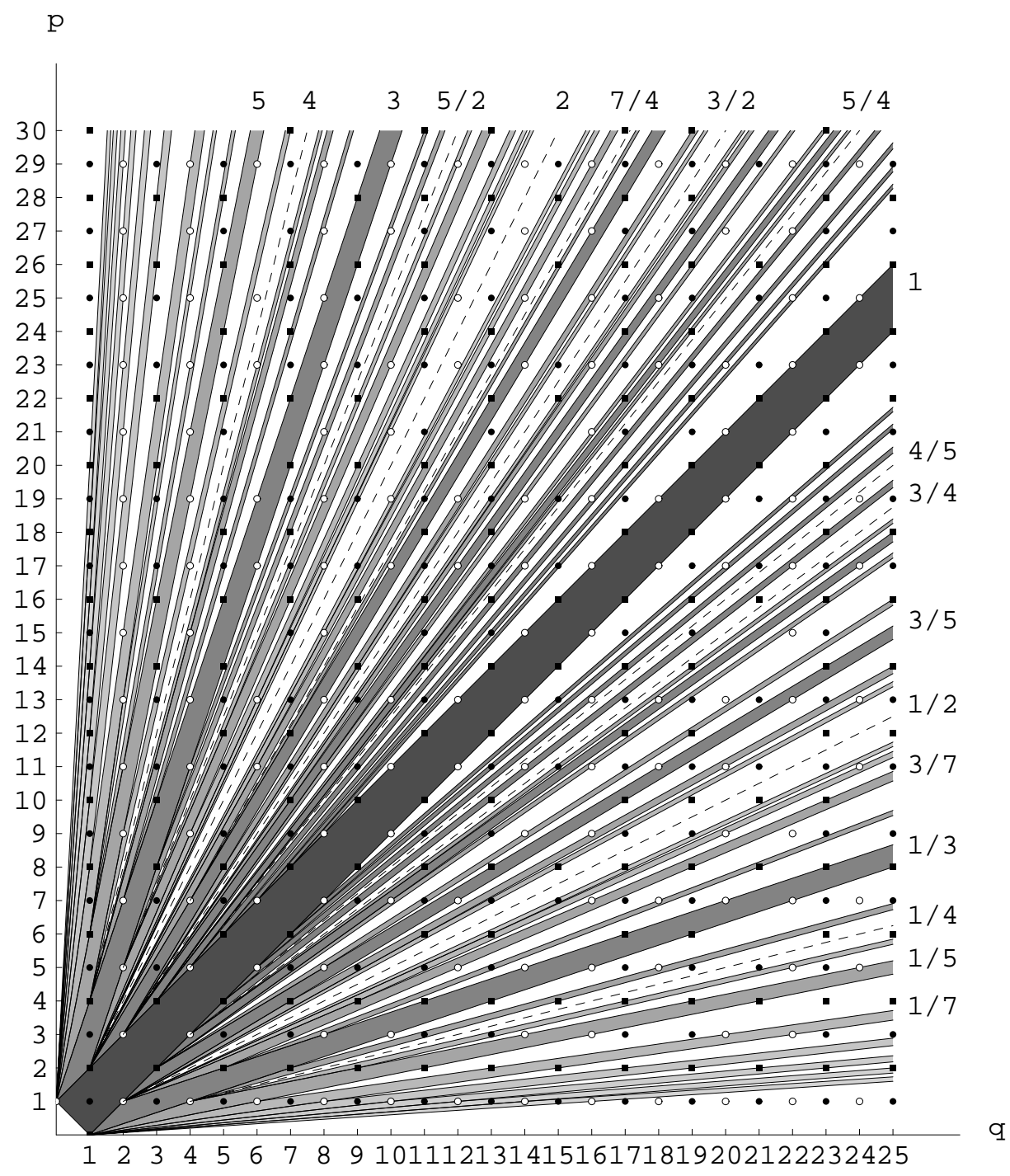

Figure 6: Odd/odd elementary diagram corresponding to filling factors with odd numerator and denominator. 


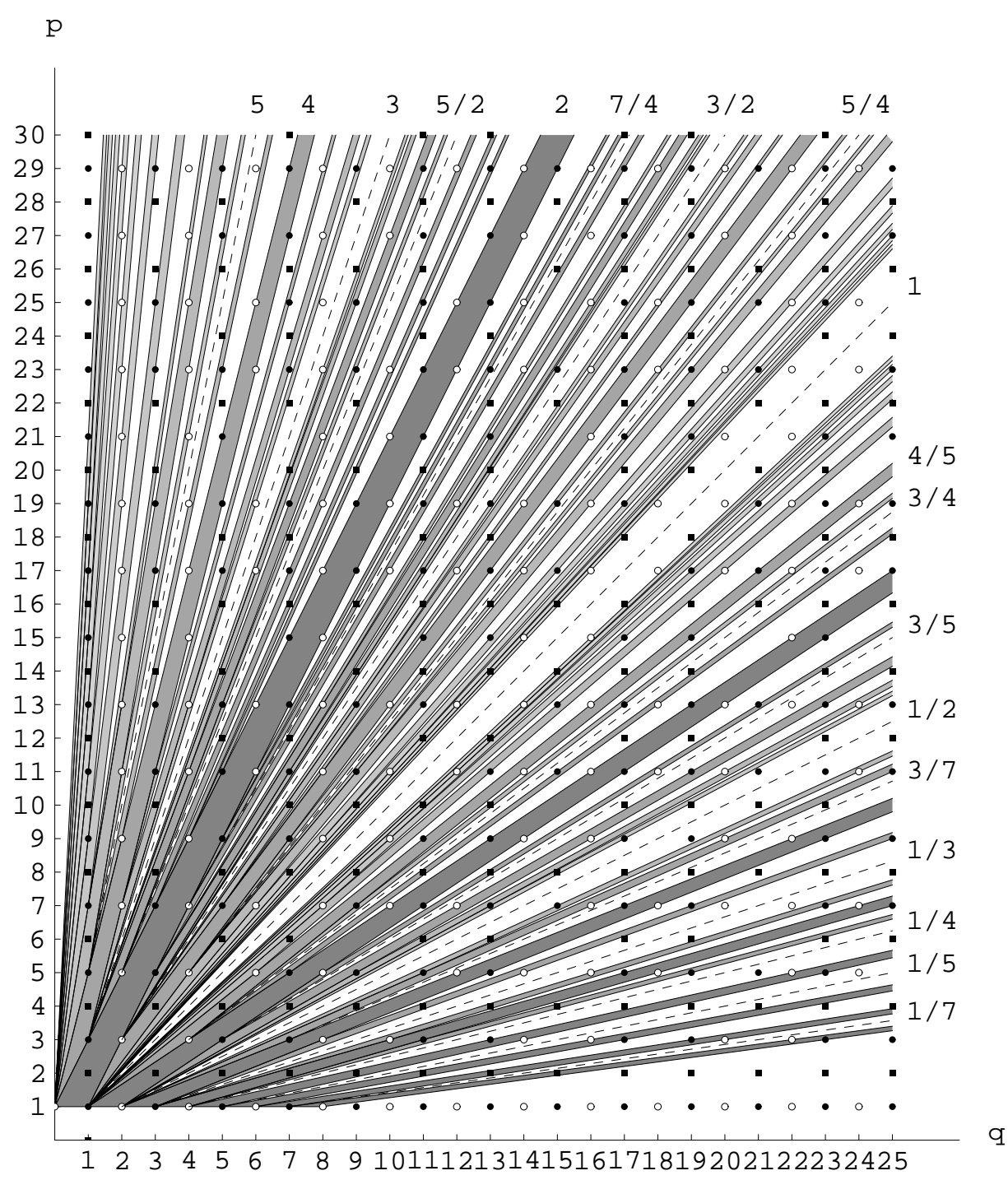

Figure 7: Even/odd elementary diagram corresponding to filling factors with even numerator and odd denominator. This diagram is related to fig. 1 through the symmetry around the $p=q$ axis. 